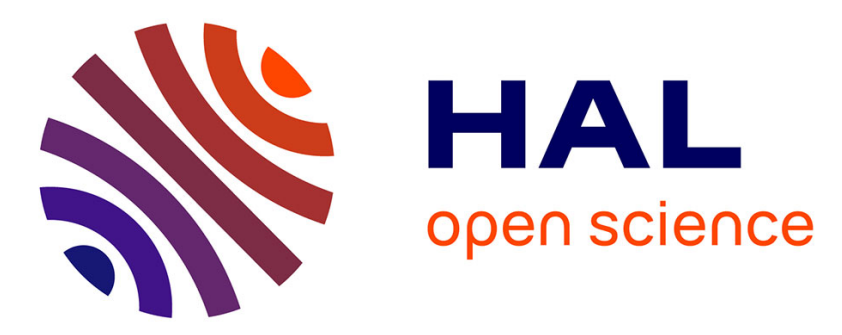

\title{
Simplified GNSS Fusion-based Train Positioning System and its Diagnosis
}

Heekwon No, Jérémy Vezinet, Carl Milner

\section{To cite this version:}

Heekwon No, Jérémy Vezinet, Carl Milner. Simplified GNSS Fusion-based Train Positioning System and its Diagnosis. ION GNSS+ 2019, 32nd International Technical Meeting of the Satellite Division of The Institute of Navigation, Sep 2019, Miami, United States. pp.3033-3044, 10.33012/2019.16967 . hal-02370234

\section{HAL Id: hal-02370234 \\ https://hal-enac.archives-ouvertes.fr/hal-02370234}

Submitted on 19 Nov 2019

HAL is a multi-disciplinary open access archive for the deposit and dissemination of scientific research documents, whether they are published or not. The documents may come from teaching and research institutions in France or abroad, or from public or private research centers.
L'archive ouverte pluridisciplinaire HAL, est destinée au dépôt et à la diffusion de documents scientifiques de niveau recherche, publiés ou non, émanant des établissements d'enseignement et de recherche français ou étrangers, des laboratoires publics ou privés. 


\title{
Simplified GNSS Fusion-based Train Positioning System and its Diagnosis
}

\author{
Heekwon No, Jérémy Vezinet, Carl Milner, ENAC, Université de Toulouse
}

\section{BIOGRAPHY (IES)}

Heekwon No is a postdoctoral researcher in the Ecole Nationale de l'Aviation Civile (ENAC), France. He received B.S. and Ph.D. degrees from the School of Mechanical and Aerospace Engineering at Seoul National University, South Korea. His research interests are the multi-sensor fusion based navigation, guidance and control systemof the unmanned aerial or ground vehicle.

Jérémy VEZINET graduated as an electronics engineer in 2010 and obtained his PhD in 2011 on multi-sensor hybridization from the ENAC (French National School for Civil Aviation) in Toulouse, France. Since 2011, he is a researcher and worked on multisensorhybridization research activities. He is now currently working on the use of GNSS for railway applications.

Dr Carl Milner is an Assistant Professor within the Telecom Lab at the Ecole Nationale de l'Aviation Civile. He has a masters degree in Mathematics from the University of Warwick, a PhD in Geomatics from Imperial College London and has completed the graduate trainee programme at the European Space Agency. His research interests include GNSS augmentation systems, integrity monitoring, air navigation and applied mathematics.

\section{ABSTRACT}

In this paper, two simple GNSS-based positioning methods are proposed and their diagnostic functions for GNSS failure are tested. Firstly, the odometer-based method, which are proposed in our previous research [1], is concretized to be implemented for general cases. This method detects faults in the GNSS solution due to satellite failure or local effects using both odometry and track geometry of the onboard system. It enables to monitor all three-dimensional solution error so that higher sensitivity for the fault detection can be achieved. Secondly, single-axis accelerometer-based approach is newly proposed. Positioning architecture of this method is designed in traveling distance domain with the configuration of single-axis accelerometer installed along the forward direction. The diagnosis of GNSS signal can be done easily in one-dimensional space. Therefore, the latter method is expected to give greater sensitivity to detect GNSS failures while maintaining a relatively simple architecture. Both methods are tested in simulation, and their abilities for detecting fault in GNSS signal are investigated and compared.

\section{INTRODUCTION}

Stakeholders in European railways are interested in the introduction of a GNSS-based positioning system for trains. GNSS is successfully implemented in the aeronautical domain. However, its introduction to rail faces an entirely different challenge. Firstly, its stringent safety requirement, set to a hazard rate of 10-9 per hour subjected to Safety Integrity Level (SIL) 4 [2] and secondly the railway user is exposed to greater interference and severe multipath in the local environment.

The use of GNSS in the railway sector has been postulated on the notion of a virtual balise (VB). Existing railway positioning systems are based on various trackside infrastructures including physical balises (PBs) installed in groups between the tracks and odometry installed on-board. However, due to the high cost of installing and maintaining the PB, a GNSS-based VB positioning performed within on-board systemhas been proposed to reduce the role of PBs. The VB-based positioning systemworks by setting a VB point on the railway track and determining the passage of the VB point using the position solution from the GNSS receiver, replacing the existing PB. However, the train user on the ground suffers from the local effect generated by the ground environment such as terrain, buildings and tunnels. The needs for a diagnostic function of the GNSS-based positioning system arises from the possibility of failures of GNSS due to the effect of the local environment. The diagnosis can be performed by using redundant measurements from other sensors.

Fusing multiple sensors provides redundant information that is useful to improve positioning accuracy and to suppress the local effects. However, additional sensors make the assessment of integrity more challenging due to the complexity of the systemand 
various error sources which cannot be easily modeled with high confidence. Installation of additional sensors also requires more cost. Therefore, a simple and minimal approach which fits the demand and requirements of the railway is desirable.

In this paper, two simple GNSS-based positioning methods are proposed and their diagnostic functions for GNSS failure are tested. Firstly, the odometer-based method, which are proposed in our previous research, is concretized to be implemented for general cases. Secondly, single-axis accelerometer-based approach is newly proposed. The latter method is expected to give greater sensitivity to detect GNSS failures while maintaining a relatively simple positioning architecture. Both methods are tested in simulation, and their abilities for detecting fault in GNSS signal are investigated and compared.

This paper is organized as follows. Firstly, PB-based and VB-based positioning systemof train are mentioned briefly. Then two fault detection method, odometer-based method and single-axis accelerometer-based method are introduced. After that, these two methods are compared by Monte Carlo simulation, and conclusion will be followed.

\section{POSITIONING SYSTEM OF TRAIN}

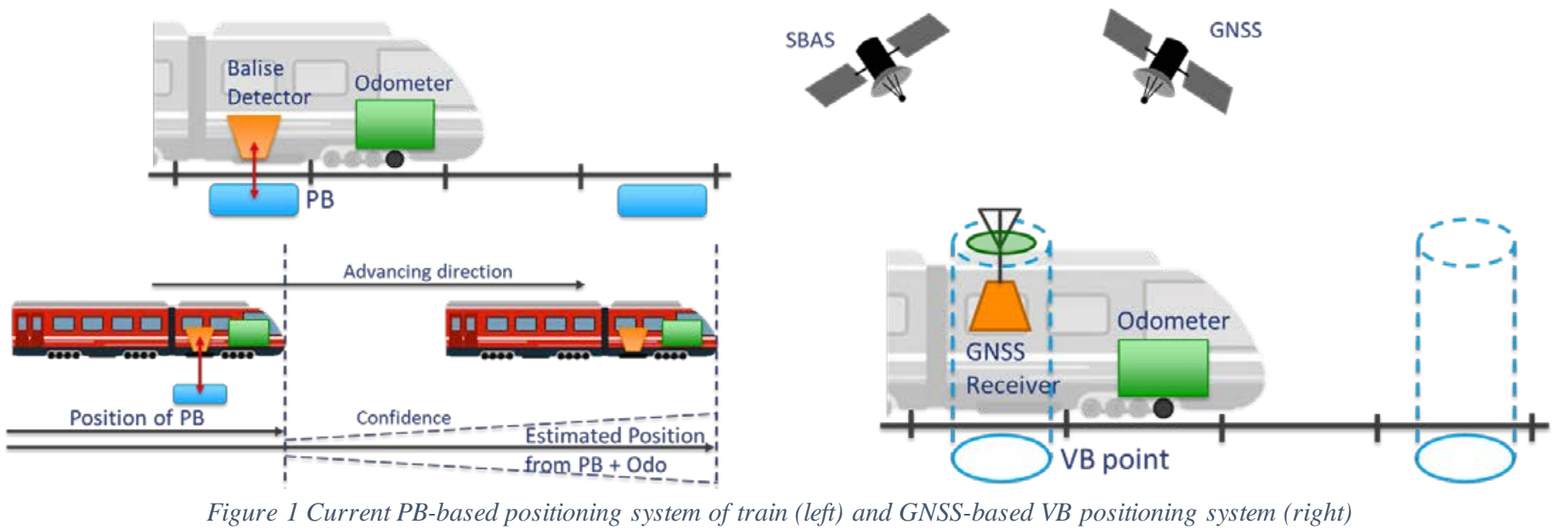

Current train positioning systemis based on PB which is installed on track side. On the train, balise detector and odometer is equipped. When the train passes over the point where the PB is installed, the balise detector receives a telegram message which contains the identification number of detected balise. From this message the train determines its current position. The train propagates its position by using measurements from the odometer when it located between balises (Figure 1). On the other hand, the concept of GNSSbased VB is proposed to reduce the role of PBs because of its cost for installation and maintenance. VB points are set on the track instead of installation of PB. When position solution of GNSS receiver, which is installed on the train, passes a VB point, the onboard VB system generates a telegram message which is similar to that of PB-based system. In this way, the VB-based positioning systemcan substitute PB-based system with maintaining compatibility to previous system.

The train is moving on the ground. Not only a fault of GNSS satellite but also ground objects can affect to the received ranging signal of train user and can generate positioning failure of VB system. The positioning failure of train user should be monitored and warned in order to meet the safety requirement of train system.

\section{ODOMETER-BASED METHOD}

In our previous research, the odometer-based method was proposed and tested under simplified assumptions. This method detects fault in position solution of GNSS by using odometer and track information which are embedded on the train. The concept of this method will be briefly introduced, and the details of this method can be found in [1].

Raw monitors for along-track direction, (1), cross track direction, (2), and vertical direction, (3) are defined as follows:

$$
\begin{aligned}
& q^{\Delta x}(k)=\Delta x^{\text {gnss }}(k)-\Delta x^{\text {odo }}(k) \\
& q^{\Delta y}(k)=\Delta y^{\text {gnss }}(k)-\Delta y^{\text {map }}(k)
\end{aligned}
$$


where

$$
q^{\Delta z}(k)=\Delta z^{\text {gnss }}(k)-\Delta z^{\text {map }}(k)
$$

$$
\begin{aligned}
& \Delta x^{\text {gnss }}(k)=x^{\text {gnss }}(k)-x^{\text {gnss }}(k-1) \\
& \Delta x^{\text {odo }}(k)=x^{\text {odo }}(k)-x^{\text {odo }}(k-1) \\
& \Delta y^{\text {gnss }}(k)=y^{\text {gnss }}(k)-y^{\text {gnss }}(k-1) \\
& \Delta z^{\text {gnss }}(k)=z^{\text {gnss }}(k)-z^{\text {gnss }}(k-1) \\
& \Delta y^{\text {map }}(k)=y^{\text {map }}(k)-y^{\text {map }}(k-1) \\
& \Delta z^{\text {map }}(k)=z^{\text {map }}(k)-z^{\text {map }}(k-1)
\end{aligned}
$$

$x^{\text {gnss }}, y^{\text {gnss }}$ and $z^{\text {gnss }}$ represent GNSS position solution for along-track direction, cross track direction and vertical direction, $x^{\text {odo }}$ represents traveling distance measurement from odometer, $y^{\text {map }}$ and $z^{\text {map }}$ represent cross track and vertical position from track information. These raw monitors have limited detectability for faults with slow mean rate because of its high noise level. Moving average is introduced to reduce the noise level of raw monitors as follows:

$$
\bar{q}_{N}^{\Delta x}(k)=\frac{1}{N} \sum_{m=1}^{N} q^{\Delta x}(k+m-N) \text { for along-track monitor }
$$

where $N$ represents window size of moving average. Bigger window size makes better sensitivity for slow rated fault, but it also induces later detection for fast rated fault than smaller window. Therefore, bank of monitors is constituted with raw monitor and moving averaged monitors with different window sizes for all three direction in order to achieve both of fast detection for fast rated fault and sensitivity for slow rated fault. In our previous research [1], exponentially weighted moving average (EWMA) was implemented. However, addressing threshold for EWMA monitor requires heavy computation due to time-correlated nature of GNSS range measurements. So, moving average is implemented instead of EWMA for light computation and similar performance.

We have four monitors for each of three directions, total 12 monitors. All monitors are monitored simultaneously, and if at least one monitor reaches its threshold value the detection of fault is declared. Entire architecture of odometer-based method is shown in Figure 2. In our previous research, several assumptions, such as fixed constellation of GNSS, and fixed heading of train, are made for easier feasibility analysis. In this paper, non-fixed constellation of GNSS, and arbitrary heading of train are considered in the calculation of threshold values for more realistic test environments.

Note that the odometer-based method is just for detection of fault, not for improving position accuracy of train. 


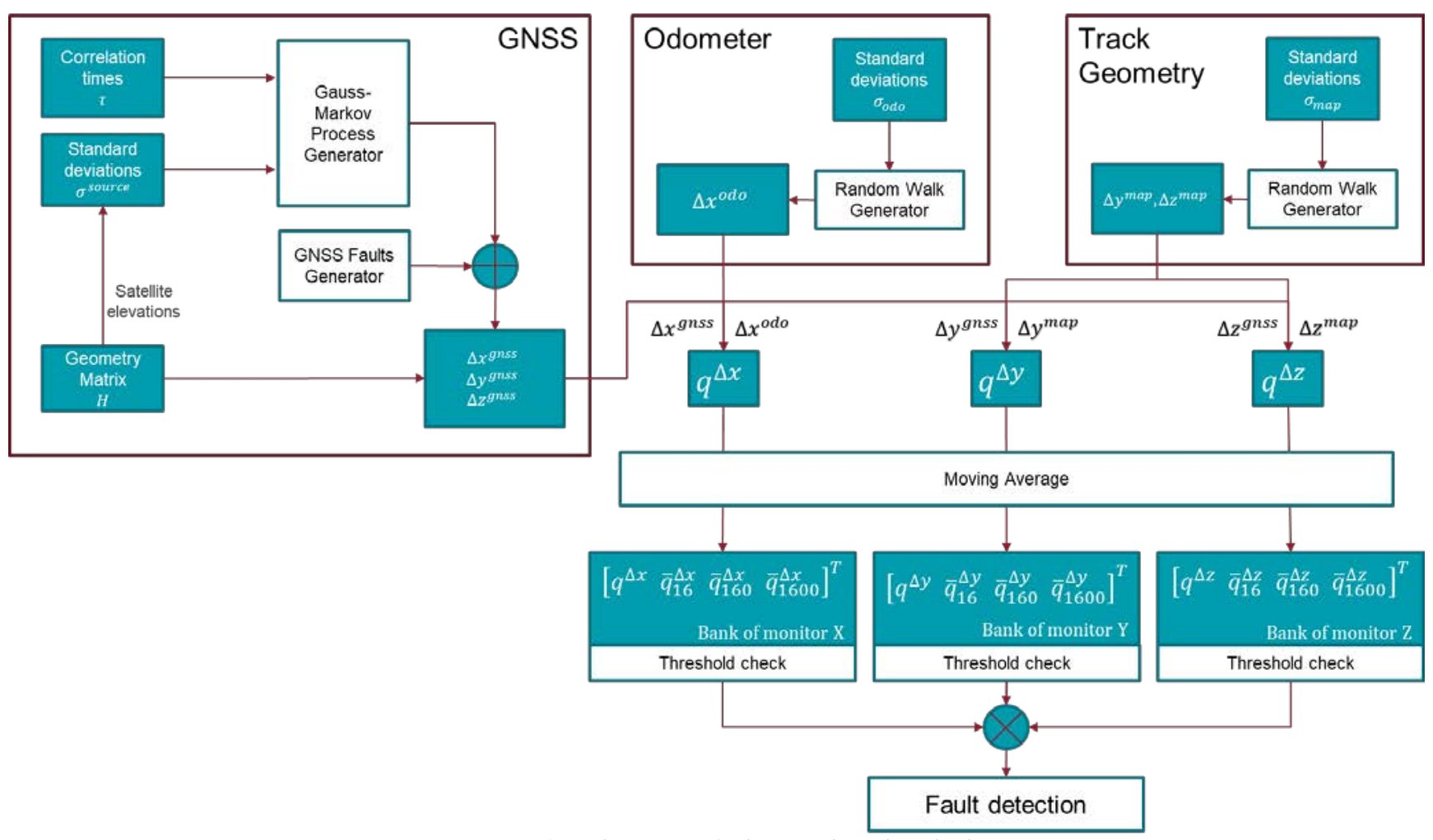

Figure 2 Architecture of odometer-based method

\section{SINGLE-AXIS ACCELEROMETER METHOD IN TRAVELING DISTANCE DOMAIN}

If we think about the moving characteristics of trains, its movement is constrained on the track. Therefore, the position of a train can be parameterized by one-dimensional traveling distance on the track (Figure 3) without considering all three-dimensional position. This is expected to simplify positioning and integrity analysis of train.

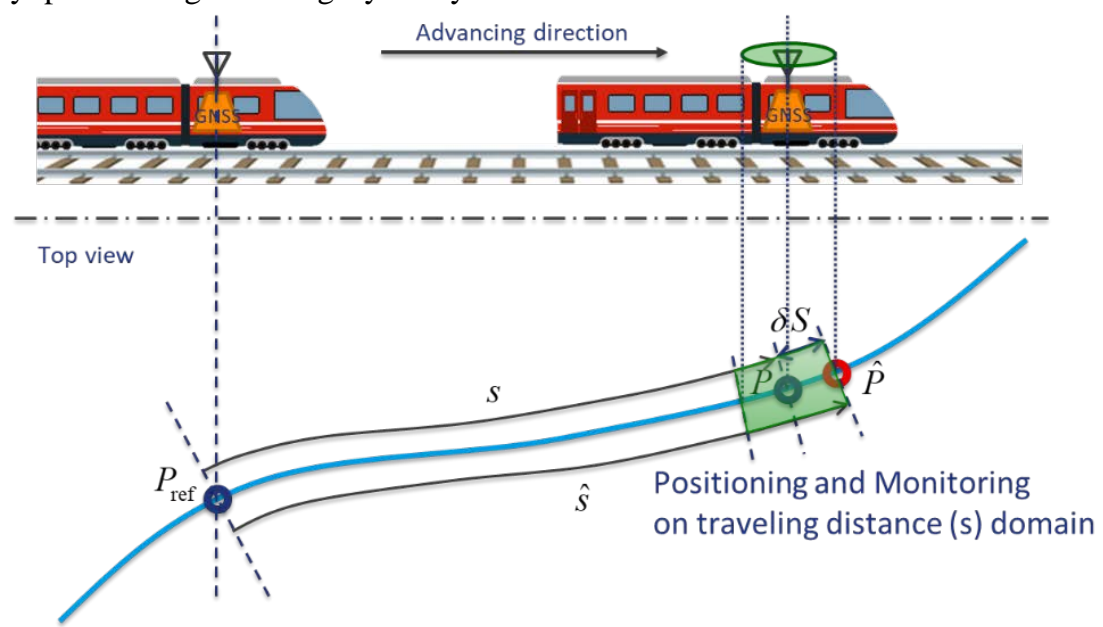

Figure 3 Parameterization of train position on traveling distance domain 
If we set a known reference position $\left(P_{\text {ref }}\right)$ on track, such as a position where a PB is installed, the traveling distance $(s)$ from reference position can represent current position $(P)$ of train. The traveling distance can be propagated by measurement of sensors, such as odometer, and its error can be estimated and corrected by measurement of GNSS.

For the propagation of traveling distance, a single-axis accelerometer installed along the direction of track is considered. The accelerometer is a self-contained sensor, and it has robustness for environmental condition compared to the odometer. The accuracy of radar odometer is sensitive to the reflective surface. Its accuracy can be degraded by rain or snow on track. The tachometer type odometer suffer from slipping or sliding when the train is accelerating or braking. Pure acceleration along forward direction is needed for the propagation of traveling distance. However, the accelerometer measures specific force which is sum of acceleration and gravity. The gravity in accelerometer measurement must be eliminated. In strapdown inertial navigation system, the gravity is eliminated by using estimated attitude information. Fortunately, inclination of track can be provided by the track geometry information. Due to the uncertainty of track geometry information the gravity cannot be eliminated completely. In that case, the odometer can be used together to suppress the effect of residual gravity.

Dynamics of the systemis simple as follows:

$$
\begin{gathered}
\dot{s}=v_{s} \\
\dot{v}_{s}=a_{s}
\end{gathered}
$$

where $v_{s}$ and $a_{s}$ are speed and acceleration along track direction. And the accelerometer measurement model is given as

$$
\begin{gathered}
f_{a}=a_{s}+g \sin \theta+b_{a 0}+b_{a 1}+w_{a} \\
\dot{b}_{a 0}=0 \\
\dot{b}_{a 1}=-\frac{1}{\tau_{a}} b_{a 1}+w_{b a 1}
\end{gathered}
$$

where $f_{a}$ is accelerometer measurement, $g$ is gravitational acceleration, $\theta$ is inclination angle, $b_{a 0}$ is constant accelerometer bias, $b_{a 1}$ is time-varying accelerometer bias, and $w_{a}$ is accelerometer noise. The time-varying bias is modeled by $1^{\text {st }}$ order Gauss-Markov process with time constant, $\tau_{a}$ and driving white noise, $w_{b a 1}$. Receiver clock bias and drift error model for tightly-coupled integration of GNSS are introduced as

$$
\begin{gathered}
\frac{d B}{d t}=\dot{B}+w_{\phi} \\
\frac{d \dot{B}}{d t}=w_{f}
\end{gathered}
$$

where $B$ and $\dot{B}$ represent receiver clock bias and drift error, $w_{\phi}$ and $w_{f}$ are driving white noise for $B$ and $\dot{B}$. Error equation in state space representation for implementation of Kalman filter is given by

$$
\begin{aligned}
& \delta x=\left[\begin{array}{llllll}
\delta s & \delta v_{s} & \delta b_{a 0} & \delta b_{a 1} & \delta B & \delta \dot{B}
\end{array}\right]^{T} \\
& \delta \dot{x}=\left[\begin{array}{cccccc}
0 & 1 & 0 & 0 & 0 & 0 \\
0 & 0 & -1 & -1 & 0 & 0 \\
0 & 0 & 0 & 0 & 0 & 0 \\
0 & 0 & 0 & -1 / \tau_{a} & 0 & 0 \\
0 & 0 & 0 & 0 & 0 & 1 \\
0 & 0 & 0 & 0 & 0 & 0
\end{array}\right] \delta x+\left[\begin{array}{ccccc}
0 & 0 & 0 & 0 & 0 \\
1 & 0 & -g & 0 & 0 \\
0 & 0 & 0 & 0 & 0 \\
0 & -1 & 0 & 0 & 0 \\
0 & 0 & 0 & -1 & 0 \\
0 & 0 & 0 & 0 & -1
\end{array}\right]\left(\begin{array}{c}
w_{a} \\
w_{b a 1} \\
\delta \theta \\
w_{\phi} \\
w_{f}
\end{array}\right)
\end{aligned}
$$

As measurements of Kalman filter, speed measurement of odometer and pseudorange measurement of GNSS are considered. The measurement equations for odometer and pseudorange are as follows:

$$
\delta v_{s}=\hat{v}_{s}-v_{\text {odo }}=\left[\begin{array}{llllll}
0 & 1 & 0 & 0 & 0 & 0
\end{array}\right] \delta x-w_{\text {odo }}
$$


where

$$
\begin{aligned}
\delta \rho^{i} & =\hat{\rho}^{i}-\rho^{i}=\left(\hat{e}^{b, i}\right)^{\mathrm{T}}\left[\begin{array}{l}
\delta x^{b} \\
\delta y^{b} \\
\delta z^{b}
\end{array}\right]+\delta B-\varepsilon_{\rho}^{i} \\
& =\left[\begin{array}{llllll}
\hat{e}_{1}^{b, i} & 0 & 0 & 0 & 1 & 0
\end{array}\right] \delta x+v_{\rho}^{i}
\end{aligned}
$$

$$
\begin{aligned}
\hat{e}^{b, i} & =\left[\begin{array}{lll}
\hat{e}_{1}^{b, i} & \hat{e}_{2}^{b, i} & \hat{e}_{3}^{b, i}
\end{array}\right]^{T}: \text { LOS vector in b-frame } \\
v_{\rho}^{i} & =\hat{e}_{2}^{b, i} \cdot \delta y^{b}+\hat{e}_{3}^{b, i} \cdot \delta z^{b}-\varepsilon_{\rho}^{i}
\end{aligned}
$$

$v_{\text {odo }}$ represents the odometer measurement, $w_{\text {odo }}$ is measurement noise of the odometer, $\rho^{i}$ represents the pseudorange measurement of i-th satellite, $\left[\begin{array}{lll}\delta x^{b} & \delta y^{b} & \delta z^{b}\end{array}\right]^{T}$ is position error vector represented in b-frame, and $\varepsilon_{\rho}^{i}$ is measurement noise of pseudorange after applying SBAS correction. In (15), along-track position error is regarded as error of traveling distance, and crosstrack and vertical position error from track geometry are absorbed to $v_{\rho}^{i}$ as components of measurement noise.

For detection of fault, innovation-based monitor is defined as follows [3]:

$$
q_{k}=\sum_{i=1}^{k} \gamma_{i}^{T} S_{i}^{-1} \gamma_{i}
$$

where

$$
\begin{aligned}
\gamma_{k} & =z_{k}-H_{k} \hat{x}_{k}^{-} \\
\gamma_{k} & \sim N\left(0, S_{k}\right) \\
S_{k} & =H_{k} P_{k}^{-} H_{k}^{T}+V_{k} \\
z_{k} & =H_{k} x_{k}+v_{k} \\
v_{k} & \sim N\left(0, V_{k}\right) \\
q_{k} & \sim \chi^{2}\left(\sum_{i=1}^{k} m(i)\right)
\end{aligned}
$$

$m(k)$ : number of measurement at k-epoch

The innovation-based monitor assumes independence of measurements over time. However, error in pseudorange measurement is

\begin{tabular}{|c|c|c|c|c|}
\hline Error & $\boldsymbol{R}(\mathbf{0})$ & $\tau$ & $R(\Delta=10 s)$ & $R(\Delta=1 s)$ \\
\hline Source & & & $R(\mathbf{0})$ & $R(\mathbf{0})$ \\
\hline Units & {$\left[m^{2}\right]$} & {$[s]$} & & \\
\hline Ionosphere & vary /w El [5] & $360 \mathrm{~s}$ & 0.9726 & 0.9972 \\
\hline Troposphere & vary /w El [5] & $1800 \mathrm{~s}$ & 0.9945 & 0.9994 \\
\hline Orbit/Clock & 0.3 & $3600 s$ & 0.9987 & 0.9997 \\
\hline User & 1.5 & $100 \mathrm{~s}$ & 0.9048 & 0.9900 \\
\hline
\end{tabular}
generally time-correlated, and it can affect to false alarm and missed detection probability. As a temporary solution for this, the update rate of pseudorange measurement is reduced to $0.1 \mathrm{~Hz}$, and standard deviation of measurement noise is exaggerated by 3times. This issue needs to be resolved carefully in future work.

\section{ERROR MODELS}

Error models for GNSS range measurements are identical to those of our previous research [1]. Modeling of GNSS errors follows previous work [4] and standard assumptions [5]. A Gauss Markov $1^{\text {st }}$ order model is assumed for each error source including ionospheric error, tropospheric error, satellite orbit and clock error and the user error consisting of nominal multipath and noise [6]. The ionospheric error and the satellite orbit and clock errors are assumed to be remaining errors after application of SBAS correction. Details of GNSS error model are shown in Table 1.

Table 1 GNSS Error model parameters 
For odometer and accelerometer, a radar type odometer and an automotive grade single-axis accelerometer are assumed. Detailed specifications are provided in Table 2.

Table 2 Sensor specifications

\begin{tabular}{cc}
\hline Odometer (radar type) & Accelerometer (automotive grade) \\
\hline $10 \mathrm{~Hz}$ measurement rate & $100 \mathrm{~Hz}$ measurement rate \\
$0.05 \mathrm{~m} / \mathrm{s}$ noise std. & 1mg noise std. \\
& Time varying bias: $1.2 \mathrm{mg}$ std. \\
& 100sec time constant \\
No constant bias \\
\hline
\end{tabular}

Embedded track geometry data is assumed to give the cross-track and vertical coordinate of the train position with $1 \mathrm{~m}$ noise considering surveying error, track deformation error, and interpolation error along the surveyed points. It is assumed that the inclination of track can be provided perfectly by track geometry information. This assumption seems too optimistic for now. However, given the smoothness of rail tracks, we think precise knowledge of inclination should be feasible. False alarm probability is set to $10^{-7}$ as a temporary value for the simulation. This value can be changed according to the requirements of rail system.

\section{SIMULATION SETUP}

To test fault detection capability of two proposed method, Monte Carlo simulation was performed. Repetition number of the simulation was $10^{4}$. In the simulation, full GPS (24 satellites) and Galileo (24 satellites) constellation were considered (Figure 4). Fault in range measurement of GNSS was assumed to be a ramp type range fault in single satellite. The tested range of ramp rate was from $0.01 \mathrm{~m} / \mathrm{s}$ to $5.0 \mathrm{~m} / \mathrm{s}$. The fault was injected to GPS satellite \#8 (pink color thick line in Figure 4) during the simulation.
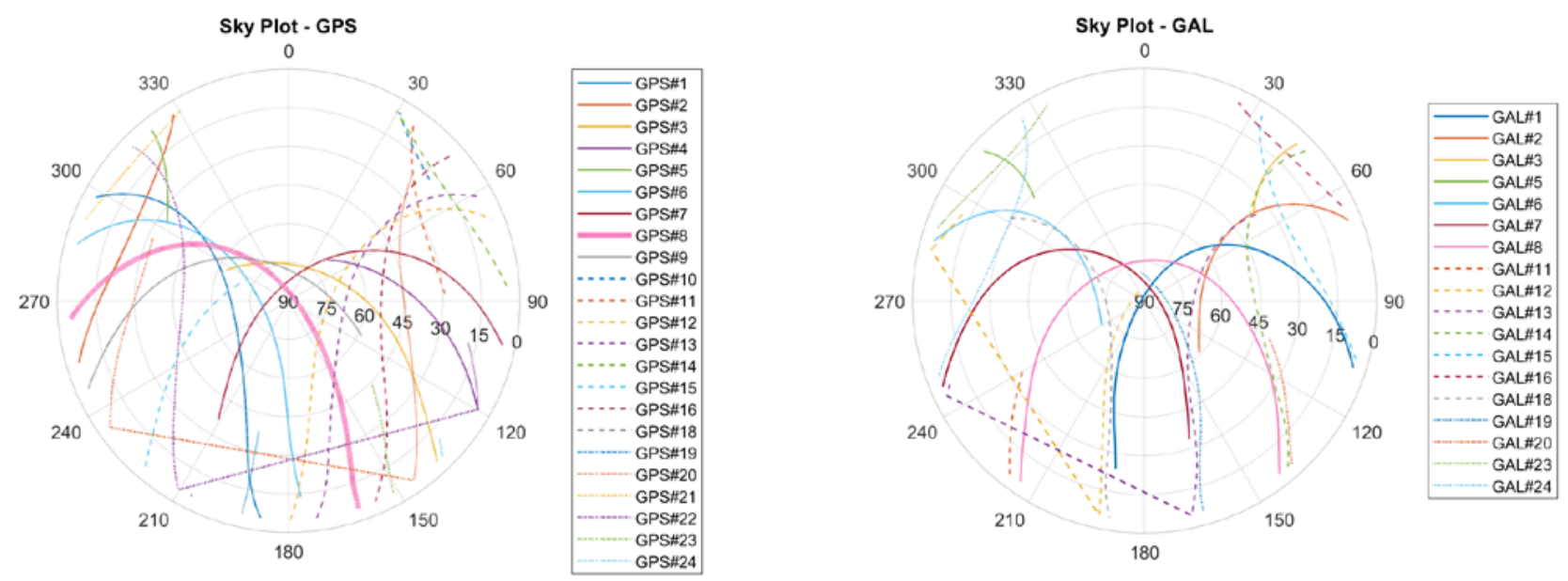

Figure 4 Skyplot of simulation, GPS (left) Galileo (right)

The duration of single run of simulation was 40000 seconds (11.1 hours). The user was assumed to move on a horizontal rectangularshaped trajectory with rounded corners (Figure 5). Its distance for one lap was $160 \mathrm{~km}$ and the user ran 5 laps with 20 m/s of speed in a single run of simulation. Initial position of user was set on Toulouse, France (latitude 43.6154 deg, longitude 1.3656 deg, height 
$524 \mathrm{~m})$. In the simulation, the range error was inserted from 15000 seconds. Time of Position failure was defined as the moment when along-track position error reaches 20 meters.

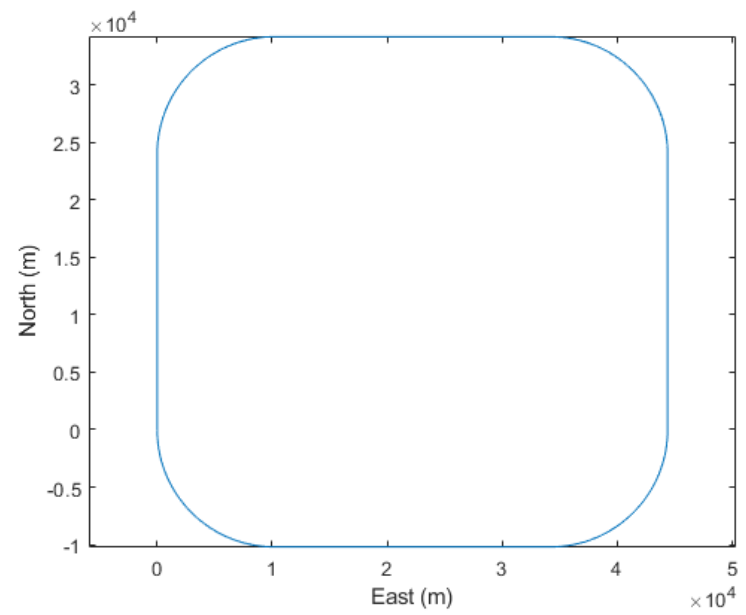

Figure 5 User trajectory of simulation

\section{SIMULATION RESULTS}

We will start to check single run results for normal and faulty condition, and then we will move to Monte Carlo simulation results for various rates of ramp fault.

Figure 6 shows single-run results of along-track position error of normal condition. 1-D KF represents the results of single-axis accelerometer-based method. The 1-D KF shows better root-mean-square (RMS) error of $0.56 \mathrm{~m}$ than $0.96 \mathrm{~m}$ of GNSS position. It is obvious that integration of accelerometer and odometer with GNSS reduces RMS position error.

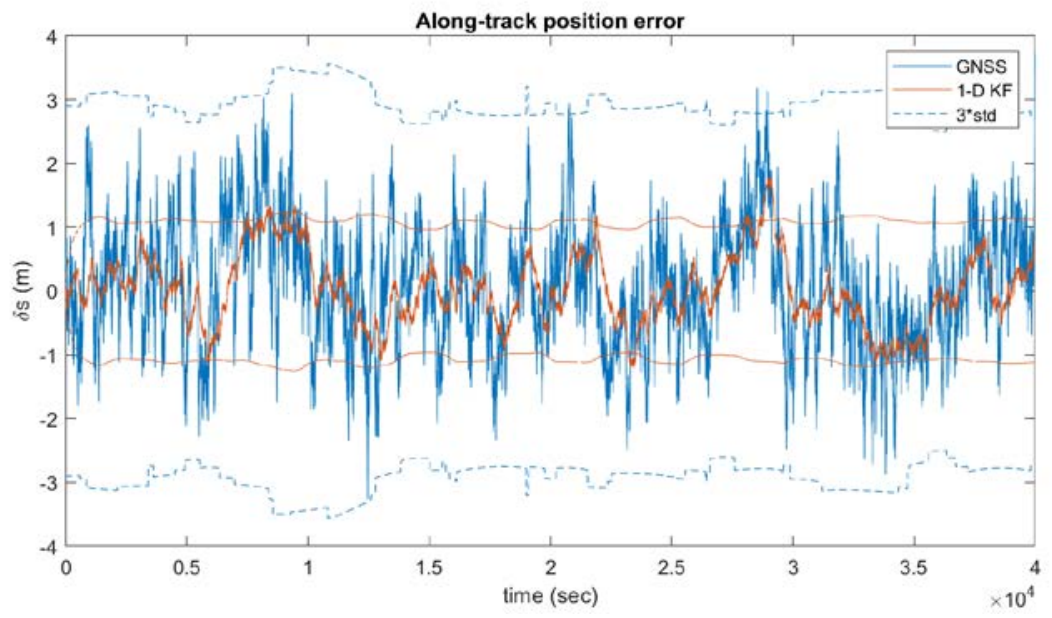

Figure 6 Along track position error results of normal condition

In single-run results of faulty condition, ramp range error of $0.1 \mathrm{~m} / \mathrm{s}$ rate is inserted. In case of faulty condition, the 1-D KF still shows better RMS error of $115.04 \mathrm{~m}$ than $169.35 \mathrm{~m}$ of GNSS, but it cannot prevent the occurrence of position failure (Figure 7). It just delays the position failure about 250 seconds from 17721 seconds to 17971 seconds. 

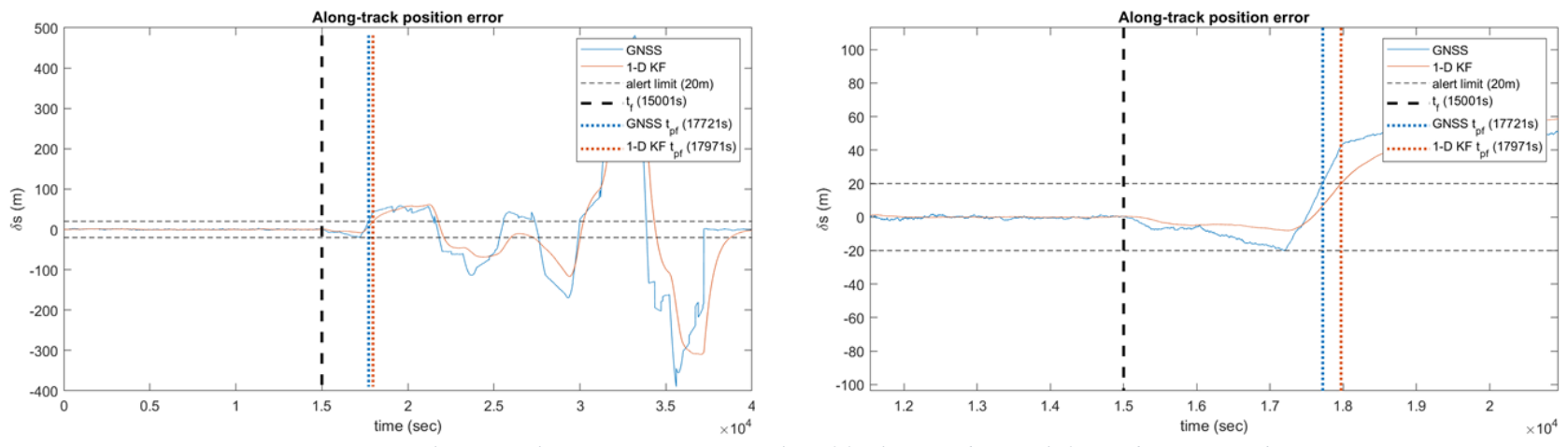

Figure 7 Along track position error results of faulty condition (left) and zoom (right)

In the Monte Carlo simulation, ramp range errors from $0.01 \mathrm{~m} / \mathrm{s}$ to $5.0 \mathrm{~m} / \mathrm{s}$ rate are tested. In the results of time of position failure, 1-D KF shows delayed time of position failure for all rates of range error compared to GNSS as shown in single-run results (Figure 8).

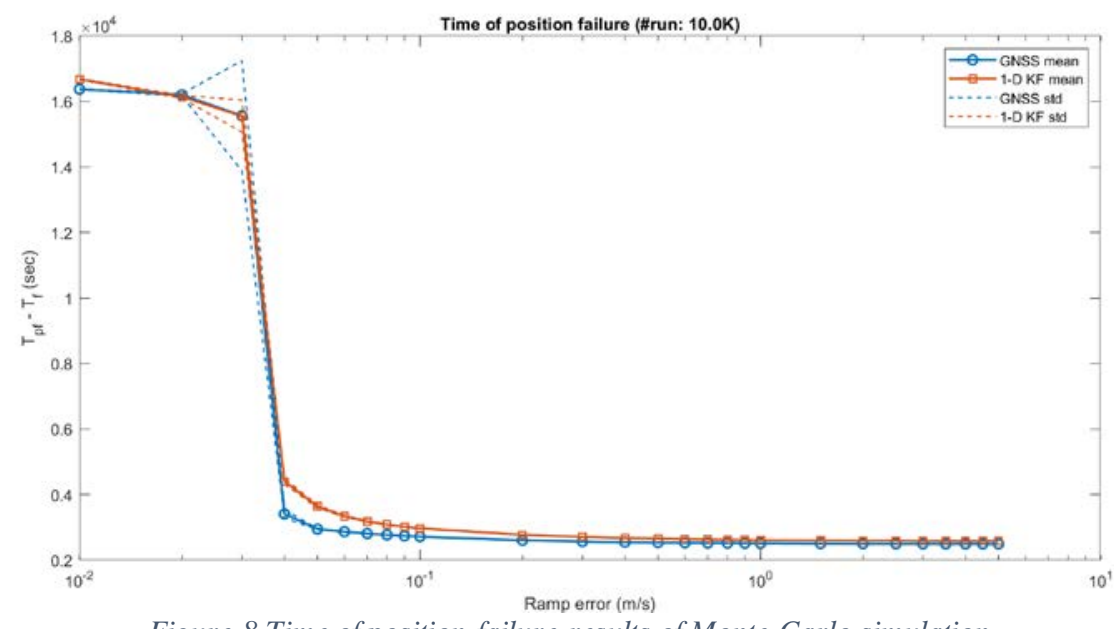

Figure 8 Time of position failure results of Monte Carlo simulation

In Figure 9, time of detection results for odometer-based method and single-axis accelerometer-based method are shown. Odo represents odometer-based method. The odometer-based method shows earlier mean detection time results than 1-D KF. Note that odometer-based method has earlier mean detection time, but it has bigger standard deviation for its detection time because it tried to detect faults using total 12 monitors, and some of its monitors, especially raw monitors, have high noise level. On the other hand, 1D KF detects faults using one innovation-based monitor, and the monitor itself is calculated by accumulation. These feature makes narrow distribution of its detection time. 


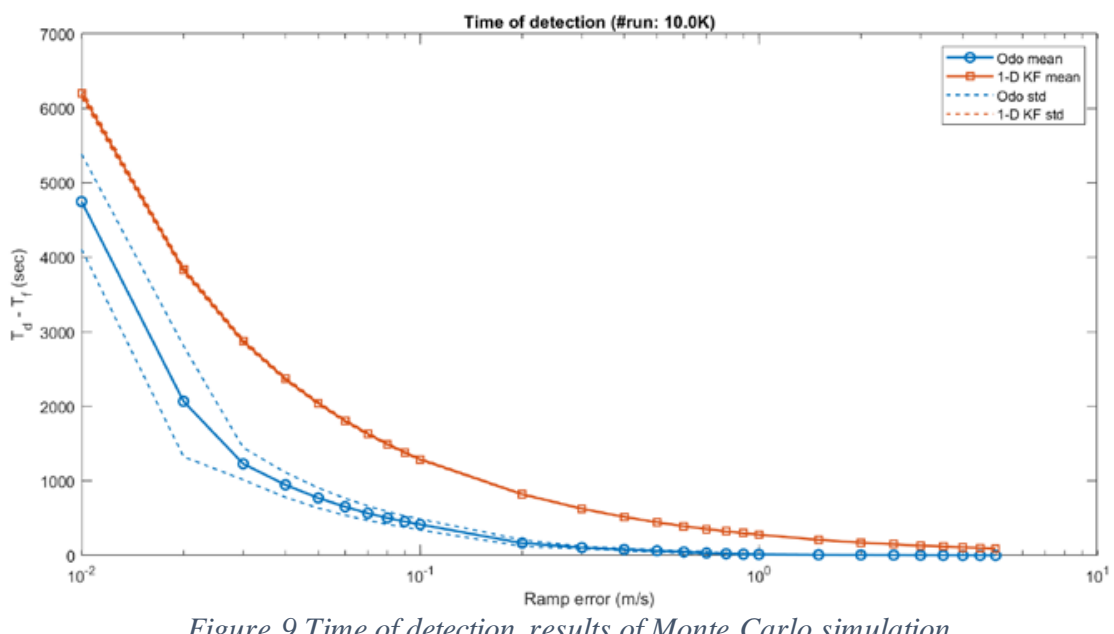

In Figure 10, probability of missed detection ( $\left.P_{\mathrm{md}}\right)$ results according to time-to-alert (TTA) are shown. TTA is defined as the time of position failure subtracted by the time of detection. Negative TTA value means the fault is detected before the position failure. In the odometer-based method, the train used GNSS position solution for its position, and TTA for odometer-based method calculated by using the time of position failure of GNSS. The results show that $P_{\mathrm{md}}$ value reaches zero on negative TTA value for both methods for all rates of ramp error. This means that the both methods succeeded to detect all faults before the position failure.

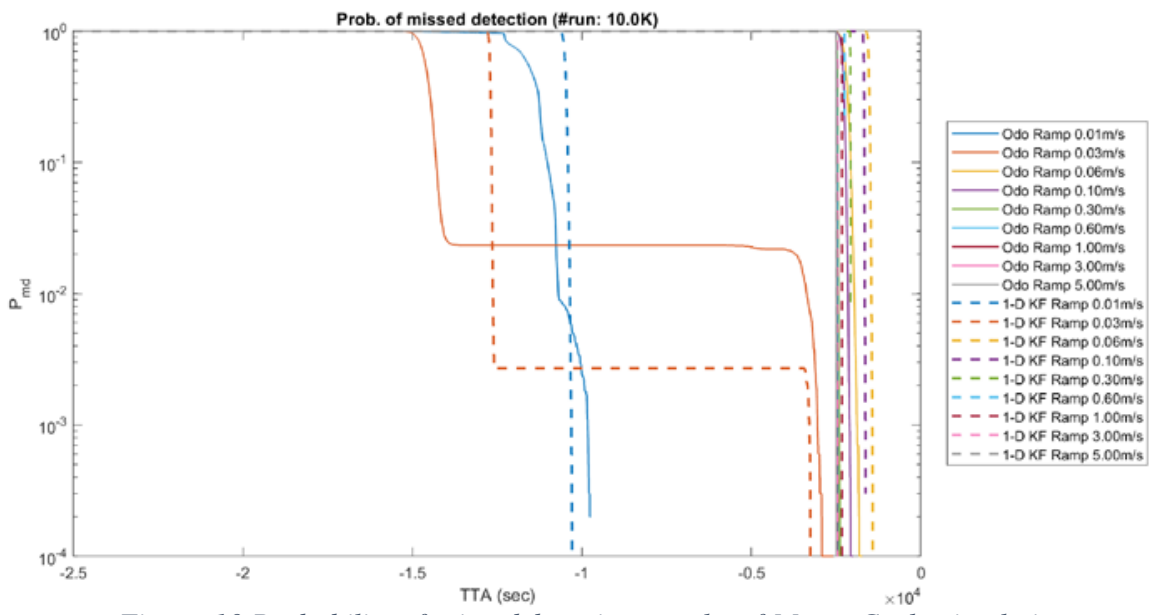

Figure 10 Probability of missed detection results of Monte Carlo simulation

Figure 11 shows mean and maximum TTA results for various rates of range error. The odometer-based method shows better (smaller) mean TTA results. However, in aspect of maximum TTA, both methods show similar results even if 1-D KF has simpler architecture for fault detection. These results were induced by narrow distribution of detection time of 1-D KF because the single-axis accelerometer method used single accumulated innovation-based monitor to detect faults. We can see potential of improved performance of single-axis accelerometer method by sophisticating its architecture by using multiple monitors with different accumulation time. This will be investigated in future work. 


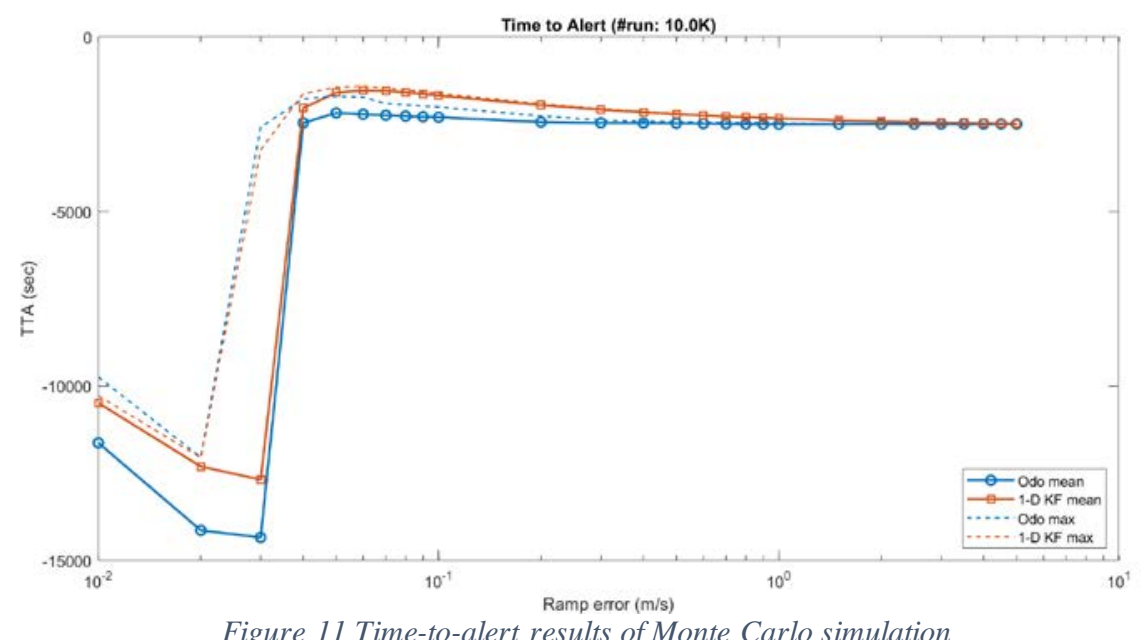

Figure 11 Time-to-alert results of Monte Carlo simulation

\section{CONCLUSION}

Skateholders in European railways are interested in the introduction of GNSS-based positioning systemfor trains. However, they are facing difficulties of the vulnerability of GNSS user on the ground and the high safety requirement of train. As a part of research for detecting failures of GNSS-based positioning system for train user, two fault detection methods are proposed and compared. The odometer-based method can detect ramp type range fault up to $0.01 \mathrm{~m} / \mathrm{s}$ mean rate using embedded odometer and track geometry information. Newly proposed single-axis accelerometer-based method handled the problem in one-dimensional traveling distance domain. It implemented the innovation-based monitor to detect fault for simpler architecture. This method has similar detection sensitivity for slow rated ramp type range fault with the odometer-based method. It shows bigger mean TTA results, but in aspect of maximum TTA, it shows similar performance because of narrow distribution of TTA. It also expected to have robust performance for environmental effects, such as weather condition or acceleration of train, because the accelerometer is not affected by those effects, but the odometer is. In the results, there is uncertainty for assumed accuracy for provided track geometry information. It will be investigated in the future work.

\section{ACKNOWLEDGMENTS}

This work has been funded by the ASTRail project. This project received funding from the Shift2Rail Joint Undertaking under the European Union’s Horizon 2020 research and innovation programme under grant agreement No 777561.

\section{REFERENCES}

1. No, H., Vezinet, J., and Milner, C., "Diagnostics of GNSS-based Virtual Balise in Railway Using Embedded Odometry and Track Geometry,” Proceedings of the 2019 International Technical Meeting of The Institute of Navigation, Reston, Virginia, January 2019, pp. 296-305.

2. Rispoli, F., Sicilano, G., and Brenna, C., “GNSS for ERTMS train localization: A step-change technology and new business model,” InsideGNSS, March-April 2017, pp. 48-54.

3. Tanil, C., Khanafseh, S., Joerger, M., and Pervan, B., “An INS Monitor to Detect GNSS Spoofers Capable of Tracking Vehicle Position,” IEEE Transactions on Aerospace and Electronic Systems, Vol. 54, No. 1, February 2018, pp. $131-143$.

4. Salos Andrés, C. D., "Integrity monitoring applied to the reception of GNSS signals in urban environments,” PhD Thesis, Institut national polytechnique, Toulouse, 2013.

5. D.-229E RTCA, "Minimum Operational Performance Standards for Global Positioning System/Satellite-Based Augmentation System Airborne Equipment (SBAS MOPS),” 2016. 
6. Pervan, B., Khanafseh, S., and Patel, J., "Test statistic auto-and cross-correlation effects on monitor false alert and missed detection probabilities," in Proceedings of the 2017 International Technical Meeting of The Institute of Navigation, Monterey, CA, 2017, pp. 562-590. 